\title{
METODOLOGÍA PARA MEDIR LA MANTENIBILIDAD PROBABILÍSTICA APLICADA A GRUPOS ELECTRÓGENOS, INDICADOR PROPUESTO PARA EVALUAR LOS RETARDOS LOGÍSTICOS, ADMINISTRATIVOS
}

\author{
Methodology to Measure Probabilistic Maintenance \\ Applied to Electric Generators Groups, Proposed Indicator \\ to Evaluate Logistics Delays, Administrative
}
César Gallegos Londoño*, Edisson Calderón Freire, Mayra Viscaíno Cuzco, Sergio Villacres Parra
Escuela Superior Politécnica de Chimborazo, Facultad de Mecánica, Escuela de Ingeniería de Mantenimiento, Riobamba (Ecuador).
*c.gallegos2009@hotmail.com

\section{$\mathbf{R}$}

La investigación se fundamenta en la evaluación global de la mantenibilidad en sistemas industriales, dividida en dos fases. La primera fase es el cálculo del indicador probabilístico de la mantenibilidad, abordando varias aristas como la marca de los equipos y los tipos de falla. Para el análisis probabilístico se utilizó la distribución de Weibull, cuyo análisis se realizó en un grupo de 91 grupos electrógenos en un lapso de diez meses. En la segunda fase se analiza los retardos logísticos y administrativos del mantenimiento que afectan a la mantenibilidad, y se delineó un nuevo indicador que permite evaluar dichos retardos. Estos procedimientos mencionados se complementan, pues el primero evalúa el tiempo de la ejecución técnica del mantenimiento y el segundo evalúa las demoras logísticas y administrativas, lo que proporcionará información útil para la toma de decisiones y la mejora continua.

Palabras claves: disponibilidad, logística de mantenimiento, mantenibilidad, mantenimiento, probabilidades

\section{A}

The research is based on the global evaluation of the maintainability in industrial systems, divided in two stages, the first one is the probabilistic indicator of maintainability calculation, addressing several edges, such as the equipment brand and the failure types. For the probabilistic analysis the Weibull distribution was used, and it was done in a group of 91 generators in a ten months period. In the second phase, the logistical and administrative delays of maintenance that affect maintainability are analyzed; therefore, a new indicator was designed to evaluate these delays. These two procedures complement each other as the first evaluates the duration of the technical execution of maintenance and the second evaluates the logistical and administrative delays, which will provide useful information for decision making and continuous improvement

Keywords: availability, maintenance logistics, maintainability, maintenance, probabilities 


\section{INTRODUCCIÓN.}

Todas las empresas dedicadas a la producción de bienes o servicios, tienen exigencias de disponibilidad de sus equipos e instalaciones. La disponibilidad de los equipos es afectada por los tiempos de parada que provocan las fallas, si se es más efectivo y eficaz al realizar acciones correctivas, se minimizarán los tiempos de intervención y se incrementará la disponibilidad $(1,2)$. El termino mantenibilidad es muy amplio, para medir la mantenibilidad se pueden usar varios indicadores, los cuales pueden ser usados en etapas diferentes, y con variados propósitos $(3,4)$. La norma UNE EN 13306:(2011), define a la mantenibilidad como la capacidad de un elemento bajo determinados contextos de utilización, para ser preservado o devuelto a un momento en el que pueda cumplir su función requerida, cuando el mantenimiento se ejecuta bajo condiciones determinadas y utilizando procedimientos y recursos establecidos $(3,5)$, este concepto relaciona a la mantenibilidad como una aptitud intrínseca o por diseño de un elemento y su facilidad de ser mantenido.

El indicador más utilizado para medir la mantenibilidad es el tiempo medio para reparar, reconocido por las siglas en inglés MTTR (mean time between failures) (6), se basa en el análisis de los históricos de los tiempos de reparación luego de ocurrir un fallo, su cálculo es relativamente sencillo, es la media de los tiempos de reparación de un equipo o un grupo de equipos (7). La norma UNE 151001:(2011), presenta un procedimiento que permite calcular varios indicadores teniendo en cuenta las condiciones de utilización de los mismos, $(2,6)$, se enfocan en el diseño del dispositivo y su facilidad de ser mantenido, evalúa criterios como: simplicidad, identificación, modularización, tribología, ergonomía, estandarización, vigilancia, etc.

La mantenibilidad puede ser revisada desde el punto de vista económico, para ello se debe analizar los costos directos e indirectos del mantenimiento, entre los costos directos se puede mencionar: la mano de obra, los materiales, los repuestos y el equipamiento utilizado (8), entre los costos indirectos se encuentran los relacionados con la pérdida de ingresos ocasionados por la parada de los equipos, conocida como lucro cesante (9), y los costos administrativos y logísticos del mantenimiento, con ello se logra identificar cual es el equipo más mantenible desde el punto de vista económico.

Otro concepto muy utilizado es la mantenibilidad probabilística, cuantificada como un indicador se lo define como la probabilidad de que un equipo, máquina o elemento que ha fallado, pueda ser recuperado y pueda seguir cumpliendo sus funciones, dentro de un intervalo de tiempo determinado, cuando se utiliza recursos e instrucciones predeterminadas $(3,5,10)$, este concepto se refiere al estudio de la mantenibilidad en términos de probabilidad, se basa en el análisis de los tiempos de reparación, conocidos universalmente con las siglas en inglés TTR (Time to Repair). Los TTR no son iguales, incluso al realizar la misma tarea de reparación en equipos idénticos, por consiguiente, son considerados variables aleatorias (5). El análisis de las variables aleatorias, debe ser descrito de forma probabilística $(8,11)$. Para el cálculo de la mantenibilidad probabilística se pueden utilizar varias distribuciones entre ellas, la distribución exponencial, la normal, log-normal, Weibull, etc. (5). La distribución más utilizada en la mantenibilidad es la de Weibull por ser muy flexible, pues posee parámetros que permiten ajustar los valores, especialmente en resultados experimentales $(8,12,13)$. La fórmula bi-paramétrica de la distribución de Weibull para la mantenibilidad viene definida por la expresión $(5,10)$ :

$$
M(t)=1-e^{-[t / \alpha]^{\beta}}
$$

Donde: $\mathrm{M}(\mathrm{t})$ es la mantenibilidad en función del tiempo, $\beta$ es el parámetro de forma, $\alpha$ parámetro de escala o vida característica, $t$ tiempo de ensayo en cualquier unidad de tiempo (14). Para el análisis de la distribución de Weibull es necesario calcular los Parámetros $\alpha$ y $\beta$, para ello se siguen los siguientes pasos:

Primer paso: Se ordenan los datos de los tiempos de reparación de menor a mayor, sin importar el orden de ocurrencia (15).

Segundo paso: Se calcula el rango de las medianas, se pueden utilizar tres fórmulas (16), las cuales se seleccionan dependiendo del tamaño de la muestra, Tabla 1. Dónde: es el rango de las 


\begin{tabular}{|c|c|}
\hline $\begin{array}{c}\text { Número de datos } \\
\text { de la muestra }\end{array}$ & Rango de las medianas \\
\hline $\mathrm{N}>50$ & $\mathrm{~m}=\frac{\mathrm{i}}{\mathrm{N}}=\frac{\sum n i}{\mathrm{~N}}$ \\
\hline $50>\mathrm{N}>20$ & $\mathrm{~m}=\frac{\mathrm{i}}{\mathrm{N}+1}$ \\
\hline $\mathrm{N}<20$ & $\mathrm{~m}=\frac{\mathrm{i}-0,3}{\mathrm{~N}+0,4}$ \\
\hline
\end{tabular}

Tabla 1. Rango de las medianas según tamaño de la muestra

\begin{tabular}{|c|c|c|c|}
\cline { 3 - 4 } \multicolumn{2}{c|}{} & Eje Y & Eje X \\
\hline $\begin{array}{c}\text { Tiempos de } \\
\text { reparación (h) }\end{array}$ & Mediana (m) & $\begin{array}{c}\text { In [In(1/1-Ran- } \\
\text { go Mediana)] }\end{array}$ & Ln (t) \\
\hline 1,02 & 0,0614 & $-2,7587$ & 0,0165 \\
\hline 2,03 & 0,1491 & $-1,8233$ & 0,7096 \\
\hline 3,05 & 0,2368 & $-1,3008$ & 1,1151 \\
\hline 4,07 & 0,3245 & $-0,9354$ & 1,4028 \\
\hline 5,08 & 0,4122 & $-0,6320$ & 1,6259 \\
\hline 8,13 & 0,5 & $-0,3665$ & 2,0959 \\
\hline 9,15 & 0,5877 & $-0,12092$ & 2,2137 \\
\hline 11 & 0,6754 & 0,1180 & 2,4144 \\
\hline 11,18 & 0,7631 & 0,3648 & 2,4144 \\
\hline 12,20 & 0,8508 & 0,6434 & 2,5014 \\
\hline 16,27 & 0,9338 & 1,0261 & 2,7891 \\
\hline
\end{tabular}

Tabla 2. Cálculo de las coordenadas de linealización muestra y $\boldsymbol{N}$ es el tamaño de la muestra.

Tercer paso: Encontrar las coordenadas según la distribución Weibull $(12,14,16)$ para la regresión lineal, se aplica:

- Para el eje (Y): $\ln [\ln (1 / 1$-Rango Mediana)]

- Para el eje (X): $\ln (\mathrm{t})$

Se grafican las coordenadas para obtener la ecuación de la recta, para este objetivo se puede utilizar una hoja electrónica como Excel o software como "Weibull ++" de Relia Soft.

Cuarto paso. Hallar los parámetros de la distribución de Weibull, para ello a la expresión de la mantenibilidad, ecuación 1, se aplica logaritmos neperianos por dos ocasiones obteniendo la ecuación [2] (14).

$$
M(t)=1-e^{-[t / \alpha]^{\beta}}
$$

$\ln \ln \{1 /[1-\mathrm{M}(\mathrm{t})]\}=\beta \ln (\mathrm{t})-\beta \ln \alpha$

La ecuación [2] se la coteja con la ecuación de la recta $(\mathrm{y}=\mathrm{bx}+\mathrm{c})$, dónde:

$$
\begin{gathered}
\mathrm{y}=\ln \{\ln [1 /(1-\mathrm{M}(\mathrm{t})]\} ; \\
\mathrm{bx}=\beta \ln (\mathrm{t}) ; \\
\mathrm{c}=-\beta \ln \alpha ;
\end{gathered}
$$

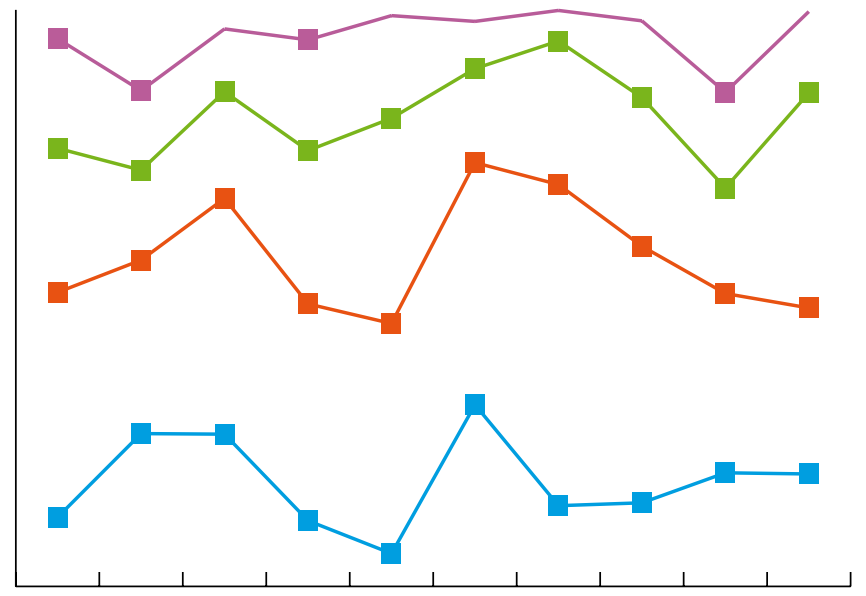

Figura 1.

El parámetro de forma $\beta$ es la pendiente de la recta de linealización, el de escala $\alpha$ se lo puede calcular al despejarlo de la ecuación [5].

$$
\alpha=\mathrm{e}^{-\mathrm{c} / \beta}
$$

Hallados los parámetros $\alpha$ y $\beta$, se calculan los valores de la mantenibilidad con la ecuación [1], se debe asignar varios tiempos de ensayo para obtener la curva de la mantenibilidad, (tabla 3).

$$
M(t)=1-e^{-[t / \alpha]^{\beta}}
$$




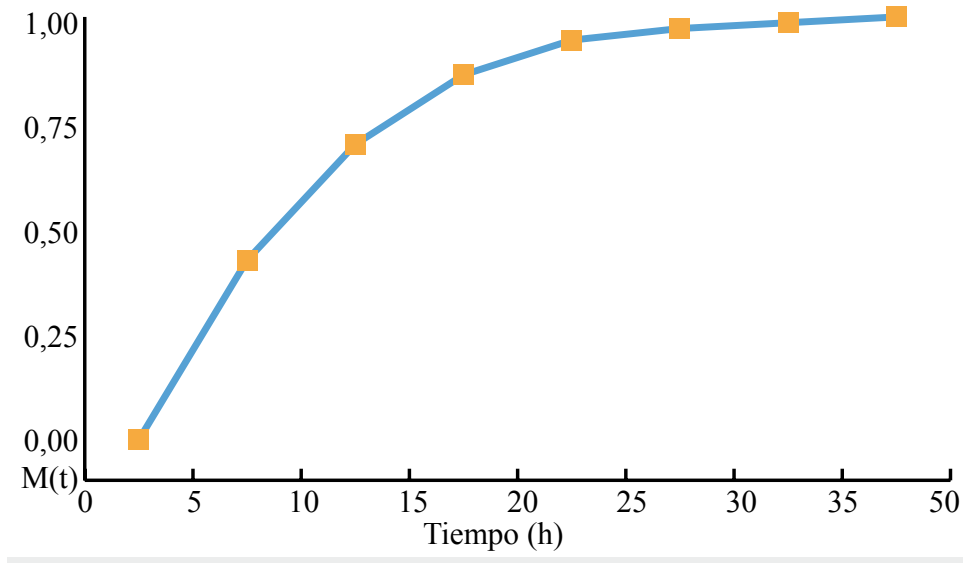

\begin{tabular}{|c|c|c|}
\hline $\begin{array}{c}\text { Tiempo } \\
\text { (horas) }\end{array}$ & Mt(t) & $\begin{array}{c}\text { Probabilidad de } \\
\text { reparación \% }\end{array}$ \\
\hline 0 & 0 & 0 \\
\hline 5 & 0,27 & 27 \\
\hline 10 & 0,58 & 58 \\
\hline 15 & 0,78 & 78 \\
\hline 20 & 0,90 & 90 \\
\hline 25 & 0,96 & 96 \\
\hline 30 & 0,98 & 98 \\
\hline 35 & 0,99 & 99 \\
\hline 50 & 1,00 & 100 \\
\hline
\end{tabular}

Tabla 3. Cálculo y curva de la Mantenibilidad

Los coeficientes de correlación $\mathrm{R}$ y de determinación, $\mathrm{R}^{2}$, establecen la prueba de bondad de ajuste para la recta de regresión (16). El coeficiente de correlación muestra la fortaleza de relación entre los datos, si se acerca a 1 confirma la dependencia lineal, mientras que el coeficiente de determinación indica el porcentaje de los puntos que están relacionados linealmente, se obtiene elevando al cuadrado el coeficiente de correlación.

Cuando ocurren los fallos, los equipos quedan en estado de indisponibilidad, este tiempo de inactividad se le conoce como Down Time (DT), el DT es la suma de los TTR (tiempo que dura la reparación neta) y el LDT ( $L o-$

\begin{tabular}{|c|l|}
\hline to & \multicolumn{1}{|c|}{ Instante en que se verifica la falla } \\
\hline $\mathbf{1}$ & Tiempo de localización del problema \\
$\mathbf{2}$ & Tiempo para el diagnóstico \\
$\mathbf{3}$ & Tiempo de desmontaje \\
$\mathbf{4}$ & Tiempo de remoción de la pieza averiada \\
$\mathbf{5}$ & Tiempo de sustitución de la pieza averiada \\
$\mathbf{6}$ & Tiempo de montaje \\
$\mathbf{7}$ & Tiempo de ajuste y pruebas \\
\hline tf & Instante de retorno a la operación \\
\hline
\end{tabular}

Tabla 4. Tiempos operativos desde la falla hasta la puesta en marcha [9].

\begin{tabular}{|l|l|}
\cline { 2 - 2 } \multicolumn{1}{c|}{} & \multicolumn{1}{c|}{ Tiempos logísticos y administrativos } \\
\hline $\mathbf{1}$ & Tiempo de notificación del problema \\
$\mathbf{2}$ & Tiempo de planificación y programación \\
$\mathbf{3}$ & Tiempo en aprobación \\
$\mathbf{4}$ & Tiempo en solicitar materiales y repuestos \\
$\mathbf{5}$ & Tiempo de compra \\
$\mathbf{6}$ & Tiempo en traslados del personal \\
$\mathbf{7}$ & Tiempo en liberación de equipos \\
\hline
\end{tabular}

Tabla 5. Tiempos logísticos y administrativos gistics delay time, tiempos de retardos logísticos y administrativos exógenos a los tiempos técnicos de la reparación) (10).

El tiempo de reparación (TTR), utilizado en el cálculo de la mantenibilidad probabilística comprende únicamente al tiempo operativo de la reparación $(5,15)$, ver tabla 4 .

Los tiempos indicados en la tabla 4, no consideran los retardos logísticos y administrativos, los cuales deben ser evaluados para un estudio global de la mantenibilidad, un detalle de los tiempos considerados como logísticos y administrativos del mantenimiento se muestran en la tabla 5.

Los aplazamientos de los trabajos de mantenimiento provocados por los retardos logísticos y administrativos, prolongan los tiempos de reparación e incluso llegan a ser superiores al tiempo de la restauración técnica del mantenimiento (1). Los retardos en la logística de mantenimiento son consecuencia de la falta de planificación, para mejorar la logística del mantenimiento se debe implementar técnicas como el Análisis de los Modos de Fallo y sus Efectos (AMFE) (17), esto logrará la identificación de tareas que corregirán futuras fallas, y se podrá determinar con antelación información relevante como: El nivel de preparación y aptitud del personal; la información técnica necesaria; equipos de 


\begin{tabular}{|c|c|c|c|c|c|c|c|c|c|c|c|c|c|c|c|c|c|c|c|}
\hline Eventos & $\mathbf{1}$ & $\mathbf{2}$ & $\mathbf{3}$ & $\mathbf{4}$ & $\mathbf{5}$ & $\mathbf{6}$ & $\mathbf{7}$ & $\mathbf{8}$ & $\mathbf{9}$ & $\mathbf{1 0}$ & $\mathbf{1 1}$ & $\mathbf{1 2}$ & $\mathbf{1 3}$ & $\mathbf{1 4}$ & $\mathbf{1 5}$ & $\mathbf{1 6}$ & $\mathbf{1 7}$ & $\mathbf{1 8}$ & $\mathbf{1 9}$ \\
\hline Mes 1 & 0,3 & 0,5 & 0,5 & 0,7 & 1,0 & 1,5 & 1,7 & 2,0 & 2,0 & 2,5 & 2,5 & 2,5 & 4,0 & 6,3 & 22,5 & - & - & - & - \\
\hline Mes 2 & 0,5 & 0,8 & 1,0 & 2,0 & 2,0 & 3,0 & 10,5 & 15,4 & 16,8 & 17,2 & 17,3 & 17,3 & 20,5 & 22,2 & 22,3 & 22,5 & 22,5 & - & - \\
\hline Mes 3 & 0,3 & 0,5 & 0,5 & 1,0 & 1,0 & 3,5 & 5,0 & 5,0 & 6,0 & 7,6 & 7,8 & 8,1 & 8,3 & 16,6 & 17,9 & 19,8 & 19,8 & 21,7 & - \\
\hline Mes 4 & 1,0 & 1,6 & 2,0 & 6,0 & 8,5 & 9,0 & 9,2 & 9,9 & 10,0 & 19,9 & 21,5 & 21,7 & 22,2 & 22,2 & 23,2 & - & - & - & - \\
\hline Mes 5 & 1,4 & 3,0 & 4,5 & 5,0 & 6,0 & 8,8 & 9,0 & 9,9 & 10,5 & 11,7 & 15,1 & 15,9 & 18,3 & 21,2 & 21,7 & 22,6 & 23,3 & - & - \\
\hline Mes 6 & 0,3 & 0,7 & 1,0 & 2,0 & 2,8 & 3,3 & 5,0 & 8,0 & 16,1 & 21,0 & 21,4 & - & - & - & - & - & - & - & - \\
\hline Mes 7 & 1,0 & 2,0 & 3,1 & 4,1 & 5,1 & 8,1 & 9,2 & 11,2 & 11,2 & 12,2 & 16,3 & - & - & - & - & - & - & - & - \\
\hline Mes 8 & 1,0 & 2,0 & 2,0 & 3,0 & 3,1 & 4,5 & 6,0 & 7,0 & 8,3 & 8,7 & 9,0 & 9,7 & 10,5 & 11,2 & 14,1 & 21,2 & 79,4 & - & - \\
\hline Mes 9 & 0,3 & 1,2 & 2,0 & 3,0 & 4,0 & 4,0 & 7,8 & 10,8 & 13,0 & 15,0 & 21,9 & 47,5 & 56,0 & 74,5 & - & - & - & - & - \\
\hline Mes 10 & 1,1 & 3,0 & 6,0 & 7,4 & 7,9 & 8,0 & 8,0 & 8,0 & 8,5 & 9,6 & 10,3 & 10,3 & 10,6 & 14,8 & 16,3 & 17,5 & 19,5 & 20,2 & 24,0 \\
\hline
\end{tabular}

Tabla 6. Lista de tiempos de reparación por mes

ensayo o de soporte; aprovisionamiento de piezas de recambio; instalaciones requeridas $(2,18,19)$. La determinación apropiada de los recursos necesarios, provocará la disminución de los retardos logísticos. El objetivo de este artículo es presentar el indicador probabilístico con un ejemplo práctico a nivel industrial visto desde varias aristas y el desarrollo de un indicador que evalúe los retardos logísticos y administrativos que afectan a la mantenibilidad global.

\section{MATERIALES Y MÉTODOS.}

Mantenibilidad probabilística. Los datos para el análisis fueron tomados de históricos de los tiempos operativos de las reparaciones registrados en órdenes de trabajo del departamento de mantenimiento, fueron recopilados por un período de diez meses, en una muestra de 91 grupos electrógenos de tres marcas distintas, tabla 6

El análisis probabilístico de la mantenibilidad se lo realizó utilizando la distribución de Weibull. Primero se calculó la mantenibilidad global, se tomó en cuenta a todos los grupos electrógenos. El análisis se lo realizó con una frecuencia mensual. Para el estudio se tomaron cuatro tiempos de referencia (probabilidad de reparación a las: 2, 10, 20 y 40 horas). En segundo lugar se realizó el cálculo probabilístico de la mantenibilidad por marca, se reorganizaron los tiempos de reparación tomando en cuenta las marcas de los equipos, el propósito de este estudio en particular fue determinar cuál de las tres marcas analizadas tiene mejores probabilidades de reparación. Para finalizar con el análisis probabilístico se hizo otra variante, se calculó la mantenibilidad por tipo de falla, se reorganizaron nuevamente los tiempos de reparación considerando ahora las fallas mecánicas, eléctricas y electrónicas, se utilizó este análisis para determinar qué tipo de fallas tienen mayor probabilidad de reparación. En todos los casos se realizó las pruebas de bondad (coeficientes de correlación y determinación) $(20,16)$.

Como se ha mencionado el tiempo de reparación de una falla se divide esencialmente en dos, el tiempo técnico de la reparación y el tiempo de los retardos logísticos y administrativos. Para el cálculo del indicador probabilístico de la mantenibilidad se utilizan los tiempos de reparación $(3,10)$, quedando fuera de este análisis los tiempos de los retardos logísticos y administrativos, a pesar de que algunos de estos tiempos son exógenos a las acciones técnicas de mantenimiento, deben ser evaluados pues afectan directamente a la disponibilidad operacional de los equipos (21).

Los retardos logísticos y administrativos descritos en la tabla 5 son de índole variada, algunos de sus valores pueden variar desproporcionadamente dependiendo de factores externos al mantenimiento como: estrategias gerenciales, mercados externos, negociaciones con proveedores de materiales y repuestos, etc. Para evaluar los retardos logísticos y administrativos se propone la utilización de un algoritmo similar al utilizado en la norma española UNE 151001 (1), utilizada para la evaluación de los atributos de diseño de los dispositivos industriales. Este algoritmo sirve para evaluar varios criterios de 
diferente índole y representarlos como un único indicador numérico adimensional. A continuación se descríbela adaptación de este algoritmo para la evaluación de los retardos logísticos y administrativos:

Paso 1. Se debe evaluar cada uno de los los tiempos de retardos logísticos y administrativos descritos en la tabla 5, se valora cada tiempo en una escala de 0 a 4 (Ti), los criterios para la evaluación son: Para tiempos inferiores a una hora la calificación es 1; si el tiempo está entre 1 y 8 horas la calificación es 2 ; si varía entre 8 y 24 horas la calificación es tres; finalmente si es mayor a 24 horas la calificación es 4 , las valoraciones se las ubica en la columna 1, ver tabla 7 .

Paso 2. Se evalúa también en una escala de 0 a 4 la importancia para la mantenibilidad de cada tiempo descrito, (PGi), se colocaron los valores en la columna 2.

Paso 3. Se calcula el porcentaje del peso de la importancia para la mantenibilidad, dividiendo la evaluación de cada importancia para la sumatoria de las evaluaciones (PGi/sPGi), se colocaron los valores en la columna 3.

Paso 4. Para el cálculo del peso se multiplica la evaluación de cada tiempo (columna 1) por el porcentaje del peso (Ti x PGi/LPGi) (columna 3), se colocan los valores en la columna 4.

Paso 5. Finalmente se suma los pesos de cada tiempo analizado y se obtiene el indicador total de los tiempos logísticos y administrativos. El indicador fluctuará entre 0 y 4 siendo 0 el mejor indicador y cuatro la peor calificación, ver la tabla 7. Se recomienda realizar una interpretación gráfica tipo radar para analizar fácilmente los resultados. Se propone una frecuencia de cálculo semestral o anual.

\section{RESULTADOS}

Mantenibilidad Probabilística: En la figura 1, se observa el cálculo mensual de la mantenibilidad de los 91 grupos electrógenos estudiados por 10 meses. Para el análisis y comparación de los resultados de las probabilidades se usaron cuatro tiempos de prueba, $(2 \mathrm{~h}, 10 \mathrm{~h}, 20 \mathrm{~h}$ y $40 \mathrm{~h})$. Se tomó la media de las probabilidades mensuales para tener un punto de base de comparación, se obtuvieron los siguientes resultados: para un tiempo de 2 horas la probabilidad de reparación es del $21 \%$, para 10 horas del $61 \%$, para 20 horas el $83 \%$ y para 40 horas la probabilidad de reparación del 95\%. Los índices de correlación y determinación son altos $\mathrm{R}=0,98$ y $\mathrm{R} 2=0,96$.

Un segundo estudio de la mantenibilidad probabilística fue analizar las probabilidades de reparación para las tres marcas de equipos, se evaluaron los tiempos de referencia 2, 10, 20 y 40 horas En el esquema de la figura 2, se puede identificar claramente la marca con mayores probabilidades de reparación Los índices de correlación y determinación son altos $\mathrm{R}_{\text {(Caterpillar) }}=097 \mathrm{y} \mathrm{R}^{2}$ (Caterpillar) $=0,97 ; \mathrm{R}_{\text {(MTU) }}=097 \mathrm{y} \mathrm{R}_{(\mathrm{MTU})}^{2}=0,97$; $\mathrm{R}_{\text {(Cummins) }}=099 \mathrm{y} \mathrm{R}_{\text {(Cummins) }}^{2}=0,97$.

\begin{tabular}{|c|c|c|c|c|c|c|c|}
\hline & \multirow{3}{*}{$\begin{array}{c}\text { Demora en tiempos logísticos } \\
\text { y administrativos }\end{array}$} & \multicolumn{2}{|c|}{$\begin{array}{l}\text { Evaluación } \\
\text { de tiempos }\end{array}$} & $\begin{array}{c}\text { Importancia } \\
\text { para la } \\
\text { mantenibilidad }\end{array}$ & $\begin{array}{l}\text { Cálculo del } \\
\text { peso } \%\end{array}$ & \multicolumn{2}{|c|}{ Peso del tiempo } \\
\hline & & \multicolumn{2}{|c|}{$\mathrm{Ti}(0-4)$} & \multirow{2}{*}{ PGi (0-4) } & \multirow{2}{*}{$\mathrm{PGi} / \sum \mathrm{PGi}$} & \multicolumn{2}{|c|}{ Ti x (Pgi/ $\sum$ PGi) } \\
\hline & & Mes 1 & Mes 10 & & & Mes 1 & Mes 10 \\
\hline 1 & Tiempo de notificación del problema & 2 & 1 & 3 & 0,15 & 0,30 & 0,15 \\
\hline 2 & Tiempo de planificación y programación & 2 & 2 & 4 & 0,20 & 0,40 & 0,4 \\
\hline 3 & Tiempo en aprobación & 2 & 1 & 3 & 0,15 & 0,30 & 0,15 \\
\hline 4 & Tiempo en solicitar materiales y repuestos & 3 & 2 & 3 & 0,15 & 0,45 & 0,3 \\
\hline 5 & Tiempos de compra & 4 & 4 & 2 & 0,10 & 0,40 & 0,4 \\
\hline 6 & Tiempos en traslados del personal & 2 & 2 & 2 & 0,10 & 0,20 & 0,2 \\
\hline 7 & Tiempo en liberación de equipos & 3 & 2 & 3 & 0,15 & 0,45 & 0,3 \\
\hline \multicolumn{2}{|r|}{ Total } & 18 & 14 & 20 & 1,00 & & \\
\hline & & \multicolumn{4}{|c|}{ Indicador de tiempos logísticos y administrativos } & 2,50 & 1,90 \\
\hline
\end{tabular}


En la Figura 3, se muestran las probabilidades de reparación por tipo de falla (mecánicas, eléctricas y electrónicas) se evaluaron para los tiempos de ensayo $(2,10,20$ y 40 horas). Los índices de correlación y determinación son altos $\mathrm{R}_{(\mathrm{F} \text { Mecánicas })}=098 \mathrm{y} \mathrm{R}_{(\mathrm{F} \text { Mecánicas })}^{2}=0,96$; $\mathrm{R}_{(\mathrm{F} \text { Eléctricas })}=099 \mathrm{y} \mathrm{R}^{2}{ }_{(\mathrm{F} \text { Eléctricas })}=0,97$; $\mathrm{R}_{(\mathrm{F} \mathrm{Electrónicas}}=097 \mathrm{y} \mathrm{R}_{\text {(F Electrónicas) }}^{2}=0,96$. Para el análisis de los tiempos logísticos se hicieron dos corridas del indicador, la medida inicial tuvo un resultado de 2.50, luego de 10 meses el indicador se valoró en 1.90, notándose una mejora notable, en la tabla 7 , y la figura 4, se presenta los resultados de los dos análisis. Las mejora en el indicador son el resultado de acciones tomadas como la mejora en la logística de comunicaciones, implementación de un software de mantenimiento y alianzas estratégicas para la adquisición de materiales. . Los datos de los tiempos analizados fueron tomados de registros históricos de mantenimiento. Posteriormente se calculó la media de cada uno de los tiempos, para iniciar el proceso de cálculo descrito.

\section{DISCUSIÓN}

Para el cálculo de la mantenibilidad general probabilística se utilizó la distribución de Weibull, se tomaron varios tiempos de ensayo $(2,10,20$ y 40 horas) para tener una visualización clara de la variación del indicador, obteniéndose medidas de tendencia (figura $\mathrm{N}^{\circ} 1$ ), el tiempo para un $100 \%$ de probabilidad de reparación superó las 40 horas manteniéndose prácticamente constante durante los 10 meses del estudio. Es necesario tomar acciones para bajar los tiempos de intervención técnica de la reparación.

Se determinó que la marca con mayor probabilidad de reparación es Caterpillar, seguida por Cummins y por último MTU, se aclara que los equipos trabajan en el mismo régimen y contextos

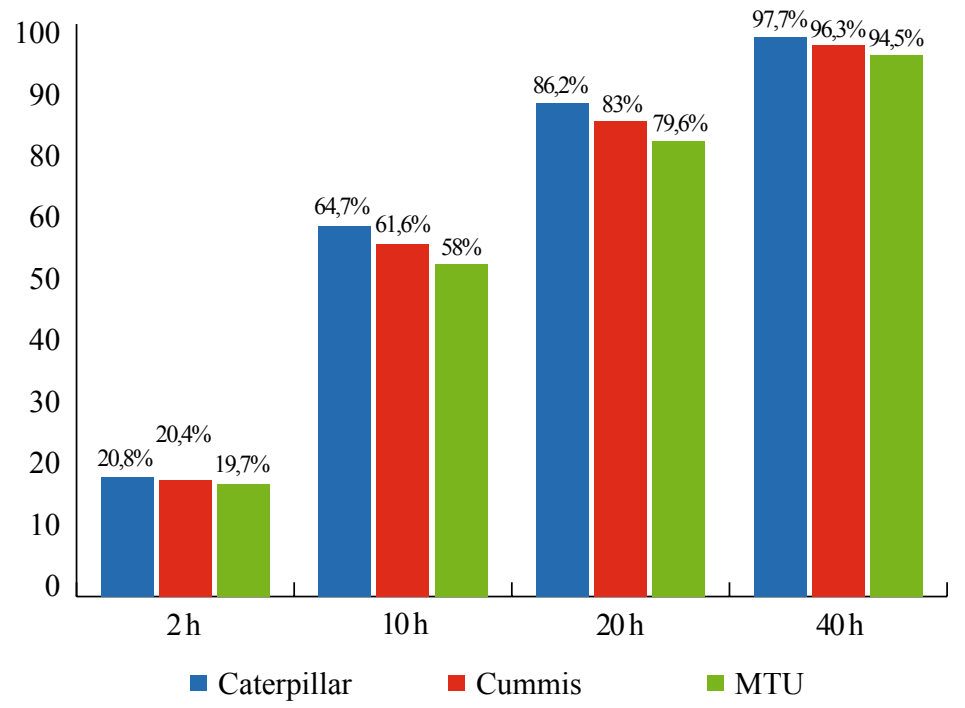

Figura 2. Probabilidades de reparación por marca

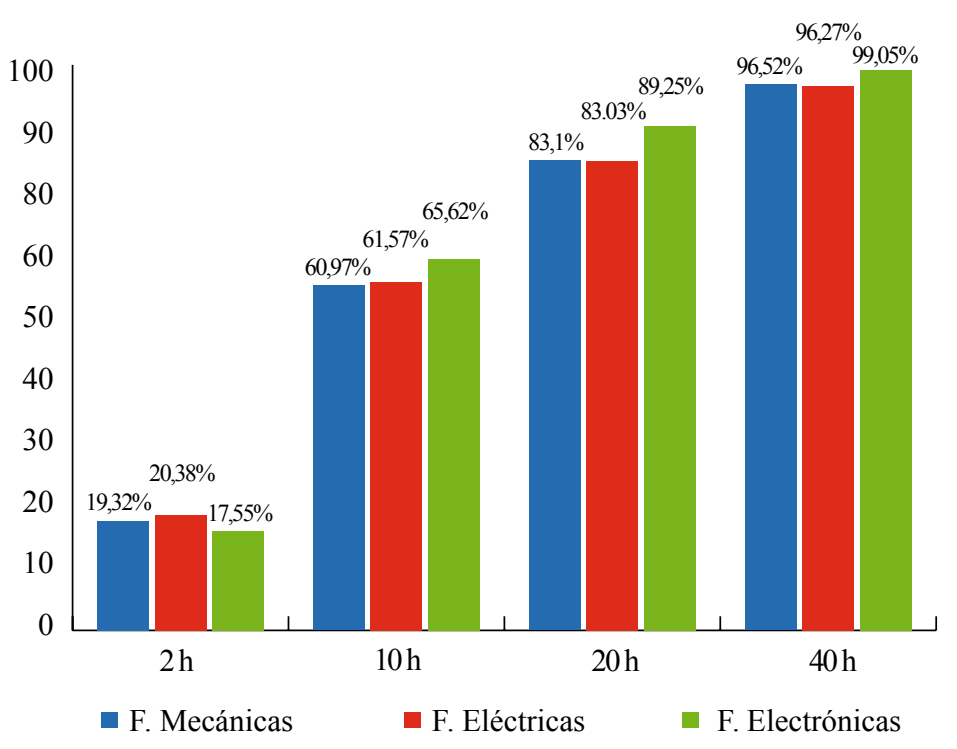

Figura 3. Probabilidades de reparación por tipo de falla

operacionales muy similares (figura 2). Además se pudo determinar qué tipo de falla tiene más probabilidades de reparación (figura 3 ).

Las probabilidades del tiempo de reparación suelen ser calculadas por equipo, sin embargo se puede analizar variantes como la marca o el tipo de falla, obteniéndose información útil para definir nuevas estrategias. Para comprobación del método se realizó adicionalmente una prueba de simulación utilizando el método Montecarlo para comprobar la validez de la distribución de Weibull. Se utilizó el siguiente algoritmo: 
Inicio

$M=$ Tamaño de ma muestra $=50$ datos

Generación aleatoria de datos entre:

TTRmin $=0,5 \mathrm{~h}$

TTRmáx $=390 \mathrm{~h}$

Cálculo de las coordenadas de linealización

Salida: Índice de Correlación $R>0,7$.

La simulación aleatoria cumplió las expectativas en el $100 \%$ de los casos conteniéndose índices de correlación superiores a 0,94 .

La norma UNE-EN 60300-3-14 Mantenimiento y Logística de mantenimiento, menciona que la logística debe ser evaluada, indica algunos aspectos para evaluar pero no propone ningún método de cálculo [22]. Si se revisa la norma UNE -EN 15341- Indicadores claves de rendimiento del mantenimiento, tiene 71 indicadores divididos en tres categorías: Económicos, Técnicos y Organizacionales [21], sin embargo muy pocos relacionados directamente con la logística de mantenimiento. El nuevo indicador propone un análisis de varios aspectos relacionados con la logística del mantenimiento, tabla 5 y los conjuga para obtener un resultado numérico adimensional.

Este trabajo se enfoca en el análisis de la mantenibilidad desde dos puntos de vista, la probabilidad técnica de reparación y los retardos logísticos y administrativos, en el caso de estudio se encontró que en el período de 10 meces no hubo un cambio significativo en la mejora de los tiempos de reparación, mientras que el análisis de los

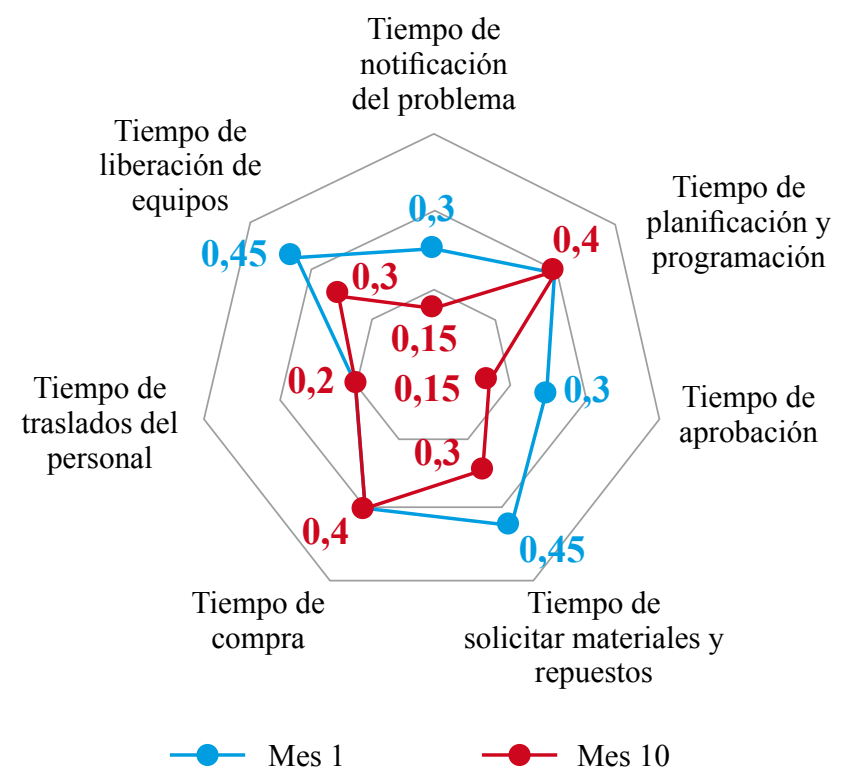

Figura 4. Indicador de retardos logísticos y administrativos, mes 1 y mes 10 tiempos logísticos y administrativos el indicador mostro un avance, pues inicio en 2,5 y terminó en 1,9 . Se recomienda que el cálculo del indicador sea semestral o anual.

\section{CONCLUSIONES}

- La mantenibilidad es un estudio muy importante a nivel de gestión, debido a que puede pronosticar la probabilidad de reparación en un tiempo determinado, el análisis probabilístico apuntó a determinar que marca de equipo tiene mayor probabilidad de reparación y de la misma manera se enfocó en determinar cuál de las fallas (mecánicas, Eléctricas y electrónicas) tienen mayores probabilidades de ser reparadas. Pudiéndose ampliar el análisis para otras características como: modelos de máquinas, áreas o secciones de una empresa, contextos operativos, etc. De esta manera se puede obtener información muy específica para la toma de decisiones. El presente artículo ofrece una herramienta estadística que permite controlar estos parámetros y como producto indirecto aumentar la productividad de una empresa.

- Los tiempos modernos, imponen resultados precisos y es menester de los profesionales de actualidad, que cuenten con todos los materiales, insumos, repuestos y equipos necesarios para dar respuesta ágil a las actividades de mantenimiento que necesita la industria. Los correctivos realizados a una máquina deben estar documentados, para lograr dar un seguimiento y proponer posibles acciones de mejora en próximas intervenciones. Esta investigación proporciona un ejemplo práctico y un procedimiento establecido para controlar los tiempos de reparación entre cada parada. Las herramientas estadísticas y el análisis de la mantenibilidad aplicadas a grupos electrógenos, sugieren una metodología fiable para medir el tiempo de ejecución técnica. 
- La ejecución de una reparación puede ser dividida en dos partes: él tiempo técnico de la reparación y el tiempo consumido en retardos logísticos y administrativos, los tiempos técnicos que deben ser analizados con la mantenibilidad probabilística, para los retardos logísticos esta investigación propone un indicador práctico y fácil de implementar. Este indicador proporciona una información útil incluso para otros de- partamentos de una empresa, como: bodega (inventario), compras, contabilidad, etc.

- Toda gestión de mantenimiento debe ser evaluada y esta evaluación debe proporcionar información necesaria para tomar acciones correctivas respecto a la gestión. El análisis de la mantenibilidad es una herramienta muy importante para cumplir con los objetivos de mejora. Esta investigación pretende aportar ideas prácticas para futuras investigaciones.

1. AENOR UNE-EN 151001. Norma Mantenimiento Indicadores de mantenibilidad de dispositivos industriales, Madrid: AENOR, 2011.

2. Kelly A, Harris M, Gestión del Mantenimiento Indistrial. Vol 1 1ra ed. Madrid: Fundación Repsol; 1977

3. AENOR UNE-EN 13306, Norma Terminología de mantenimiento, Madrid: AENOR, 2011.

4. León.P, González V, Moreu P, Crespo A, Barberá L, Norma de mantenibilidad propuesta para la evaluación de la mantenibilidad de activos industriales.Ingeniería y gestión del mantenimiento. 2011; 3-13.

5. Kenezevic J, Mantenibilidad. Vol 9. 4ta ed. Madrid: ISDEFE, 1996.

6. Goulden E, An analytic approach to performing a mantainability demostration. IEEE Transactions on Reliability, 1990; 19-22.

7. Tabarea L, Administración moderna del mantenimiento, Vol 1, 2da ed. Sao Paulo, 2000.

8. Benitéz R, Díaz A, Carrera J. Metodología para el cálculo de la mantenibilidad. 2014; 2-6.

9. Hernández E, Anulo B, Fiallos $\mathrm{P}$, Chavez V, Método para el cálculo del costo de la indisponibilidad en procesos productivos. Perfles. 2017; 91-94.

10. Mora A, Mantenimiento estratégico para empresas industriales o de servicio. Vol 1. 1ra ed. Medellín: Ultragráficas, 2005.

11. Kenezevic j, Mantenimiento. Vol 10, 4ta ed. Madrid: ISDEFE; 1996.

12. Red temática nacional sobre seguridad de funcionamiento y calidad de servicio de sistemas productivos. Aproximaciones de la Confiabilidad Aplicaciones Prácticas. Vol 1. 1ra ed. Madrid: Ingeman, 2010.

13. Parra c, Crespo A, Métodos de análisis de fiabilidad, mantenibilidad, disponibilidad y riego, Ingecom, 2016, 23-27.

14. Tamborero J, NTP 331. Fiabilidad: la distribución de Weibull. NPT 331, Madrid: 1994.

15. Mesa D, Ortíz Y, Pinzón M, La Confiabilidad, la Disponibilidad y la Mantenibilidad, diciplinas modernas aplicadas al mantenimiento, Scientia y technica, 2ISSN 0122-1701, No 30, 2006; 1-10.

16. Zabala W, Flabilidad de Máquinas, Vol 1, 1ra ed. Riobamba: Espoch, 2004.

17. AENOR UNE-IEC 60300-3-10. Norma Guía de aplicación Mantenibilidad parte 10. Madrid: AENOR, 2007.

18. AENOR-UNE-EN 20654-4. Norma Planificación del mantenimiento y logística del mantenimiento parte 4 de 8. Madrid: AENOR, 2002.

19. Rey F. Hacia la excelencia en mntenimiento, Madrid: Tecnologías de gerencia y producción S.A.TGP-HOSHIN SL, 1996.

20. Yanez M, Gómez H, Valvuena G, Ingeniería de la confiabilidad y análisis estadístico del riesgo. Madrid: Reliability and risk management; 2004.

21. AENOR.UNE-EN 15341, Indicadores claves del mantenimiento. Madrid: AENOR; 2008. 